\title{
Antibiotic prescribing among inpatients with infectious and non-infectious indications in obstetrics and gynaecology departments at two tertiary care hospitals in Central India
}

\section{Anna Machowska}

Karolinska Institutet Department of Public Health Sciences

Kristoffer Landstedt

Karolinska Institutet Department of Public Health Sciences

Cecilia Stålsby Lundborg

Karolinska Institutet Department of Public Health Sciences

Megha Sharma ( $\triangle$ megha.sharma@ki.se)

https://orcid.org/0000-0001-9165-9393

\section{Research article}

Keywords: Antibiotic prescribing, Inpatients, Surgical prophylaxis, Bacterial infections, Obstetrics and Gynaecology, Central India

Posted Date: October 7th, 2019

DOI: https://doi.org/10.21203/rs.2.15649/v1

License: (c) (i) This work is licensed under a Creative Commons Attribution 4.0 International License. Read Full License 


\section{Abstract}

Background : Patients in departments such as obstetrics and gynaecology (OBGY) are at high risk of lifethreatening infections, thus are prescribed antibiotics extensively. Use of antibiotics leads to increasing antibiotic resistance. Antibiotic surveillance is one of the cornerstones to combat antibiotic resistance. However, hospitalbased, department specific surveillance data of prescribed antibiotics are scarce, especially in low- and middleincome countries.

Aim: To describe and compare antibiotic prescribing patterns among the inpatients at OBGY departments of two tertiary care hospitals, one teaching (TH) and one non-teaching (NTH), in Central India.

Methods: Data of all inpatients was collected manually for three years and analysed using demographics, length of hospital stay, diagnoses and prescribed antibiotics including dose, duration, and frequency and defined daily dose per 1000 inpatients were calculated. The patients were divided into, infectious and non-infectious categories and further into surgical, non-surgical and possible surgical indications. The data was coded based on the Anatomical Therapeutic Chemical classification system and the International Classification of Disease system version- 10 . Results: Of the total 5558 admitted patients, 2044 (81\%) in the TH and 2567 (85\%) in the NTH received antibiotic treatment $(p<0.001)$. In both hospitals, a majority of the patients with surgical indications were prescribed antibiotics (87\% to $100 \%$ ). Prescribing of the fixed-dose combinations of antibiotics (FDCs) and use of brand names was more common at the NTH then at the TH. A majority of the inpatients who neither had surgery nor had any confirmed bacterial infection also received antibiotic prescriptions (TH-71\%, NTH-75\%). Overall, higher DDD/1000 patients were prescribed in the TH compared to the NTH in both categories.

Conclusions: More frequent prescribing of broad-spectrum antibiotics, including FDCs and higher brand-name prescribing at the NTH compared to the $\mathrm{TH}$, is a point of concern. Antibiotics prescribed to the inpatients having non-bacterial infection indications is another point of concern and requires urgent action. Investigation of underlying reasons for prescribing antibiotics for unindicated diagnoses and the development and implementation of antibiotic stewardship programs are recommended measures to improve the prescribing of antibiotics.

\section{Background}

Antibiotics are grossly used in healthcare facilities, and its use can be quantified by surveillance of the prescriptions. Development and implementation of local prescribing guidelines based on surveillance of the prescriptions and resistance patterns is a crucial step towards combating antibiotic resistance. Despite this, the surveillance at the healthcare facilities is underperformed globally, specifically in densely populated, low- and middle-income countries (LMICs) like India.

The morbidity and mortality due to the rapid emergence of bacterial resistance with subsequent treatment failure of common bacterial infections have increased around the globe (1). Use of antibiotics, whether indicated or not, is 
a significant contributor to the development and spread of bacterial resistance (2-5)(6-8). Bacterial resistance has already reached a worrisome level and is still on the increase (5)(9). Surveillance of antibiotic use corresponding to the indications is one of the cornerstones to develop strategies to improve the use of antibiotics and to slow down the development of bacterial resistance $(2,10,11)$.

The global use of antibiotics has increased by $35 \%$ during $2000-2010$. This increase was $37 \%$ in India during 20052010 where the highest increase was reposted in broad-spectrum antibiotics such as penicillins, cephalosporins, quinolones and carbapenems (12). However, the published studies have not investigated the correlation between the choices of treatment with the indications (15-18).

The prescriptions are quantifiable and best resources to conduct the surveillance studies; however, in resourceconstrained healthcare settings, the patients' records are generally not computerised and often documented manually on the paper. Thus, lack of automated data entry at the LMICs makes the prescription surveillance studies an expensive and cumbersome process and is a significant cause of the delay in the development of contextualised antibiotic prescribing guidelines. The lack of local prescribing guidelines obstructs rational prescribing of antibiotics and lead to the development of antibiotic resistance.

The private sector is a major healthcare provider in India and constitutes $93 \%$ of all healthcare. It also plays a significant role in the overall increase in antibiotic consumption and resistance in the country (13-15). Despite this, limited studies have been conducted for the surveillance of antibiotic prescribing at Indian private healthcare sector. Few studies conducted in private settings in India presents overall high antibiotic prescribing rates $(3,16$, 17). These findings are in line with the available evidence of high use of antibiotics and the presence of various multidrug-resistant bacterial strains in the country (18).

Antibiotics are essential in high infection risk departments (where minor and major surgical procedures take place) for both, the treatment of infection and as prophylaxis for various surgery procedures, to prevent healthcare-associated infections (HAIs). Majority of the preventable maternal deaths occur in LMICs, including India (19). However, studies presenting the antibiotic prescribing pattern at these high-risk departments in India, such as Obstetrics and Gynaecology (OBGY) department, are scarce.

\section{Methods}


The present study followed an observational, cross-sectional, prospective design. The data was collected for three years between 2008 and 2011, from the OBGY departments in one TH and one NTH in Ujjain district of Central India. Both study hospitals are private sector tertiary care hospitals, though both differ in mode of providing services.

The TH is located in a rural area and provides all medical services free of charge. All medicines were purchased by the hospital management and were dispensed free of cost to all patients visiting the $\mathrm{TH}$, during the time of the study. Participation in academic activities is a part of the routine at the TH. The NTH is located in an urban area of Ujjain city where the patients are charged for the medical consultancy, hospital stay and have to purchase the prescribed medicines out of the pocket during their hospital stay. Management of the TH does not allow consultants to have a private practice, whereas most of the consultants visiting the NTH had their private clinics. The salary structure differed between the hospitals, as in the TH the physicians/ surgeons receive a fixed salary while the payment of physicians/ surgeons in the NTH is mainly based on the number of patients' admissions made in the hospital.

\section{Data Collection and study population}

The data collection method is described elsewhere in detail (17). In brief, the data of antibiotic prescribing practices was collected manually for three years in the two study hospitals. A customised form was developed and used for comprehensive data collection, which included patient's demographics, admission, and discharge dates, and details of prescribed antibiotics during the stay and at discharge, such as the name of antibiotic, dose, duration and frequency. All diagnoses (indications), as written in the patients' file at the time of discharge, were recorded in the form. The nurses working routinely in the department were trained to collect the information from the patients' record files in the paper forms. The filled forms were collected from the wards after the patient's discharge.

\section{Inclusion criteria}

Female patients who spent at least one night in the OBGY departments were at least 15 years old were included for the data analysis $(20,21)$ (Figure 1).

Data management and analysis 
The diagnoses were grouped according to the International Statistical Classification of Disease and Related Health Problems-Tenth Revision (ICD-10) (22). Some patients had a surgical procedure (with an unspecified site) or a complaint written in the diagnoses column. These diagnoses do not have any ICD-10 code and thus, were abbreviated by the authors, for example, AH for abdominal hysterectomy. Diagnoses, as assigned by the treating consultant and registered in the patients' file, were considered as final and were not validated externally.

Based on the stated presence or absence of infections in the records, the patients were divided into, infectious and non-infectious categories, respectively. The infectious diagnoses category comprised of all patients with confirmed or suspected infectious diseases including bacterial, viral and fungal infections or having the clinical signs of infection such as fever. The non-infectious diagnoses category comprised of all patients who had no documented or clinical signs of any infection. Each of these infectious and non-infectious categories was further divided into three diagnoses groups; surgical, non-surgical and possible surgical (Figure 1). Patients who had an indicated or confirmed surgery status was assigned to the surgical patient's group. Patients who had no indication for surgery were included in the non-surgical group. Patients that had a diagnosis where a surgical procedure was indicated but confirmed status of surgery conducted was not specified were included in the possible surgical patient's group.

Data analysis was performed anonymously using unique patient codes assigned after data collection. The prescribed antibiotics were categorised in Anatomical Therapeutic Chemical (ATC) classification codes; according to the WHO Collaborating Centre for Drug Statistics Methodology (WHOCC). Antibiotics could be prescribed both by generic names and trade names in the study hospitals. Generic names were entered for all antibiotics prescribed by brand names to perform analysis to use the WHO methodology. The Defined Daily Doses (DDD), as suggested by WHOCC, was used as a measuring unit for the prescribed dose (23).

Adherence to the National List of Essential Medicines of India (NLEMI) was analysed and compared between the hospitals (24). The NLEMI is customised for the national context; for example; oral metronidazole is coded as an antiprotozoal (P01) agent by WHOCC while it is an antibacterial used for anaerobic infections in the NLEMI. Recommendations of NLEMI were followed in the present study (24). For the new FDCs of antibiotics for which ATC codes are not assigned by the WHOCC, the earlier generated codes, J01RA*, were used (17). 
Data were analysed using Excel, STATA software version 13.1 (Stata Corp., College Station, Texas, USA) and SPSS Statistics version 22 (SPSS Inc, Chicago, IL, USA). For continuous values sum, mean and standard deviations were calculated. For the categorical values, frequency and percentage were calculated. Decimals were rounded off to the nearest number. The independent samples t-test was used for comparison of continuous variables since the variables followed a normal distribution at each hospital. For comparisons of categorical values, Pearson chi-square was used. P-values $\leq 0.001$ were considered significant, according to Bonferroni's correction.

\section{Results}

Of total 6208 patients admitted in both hospitals, 5558 patients fulfilled the inclusion criteria, i.e., 2539 (46\%) patients in the TH and 3019 (54\%) in the NTH (Figure 1). A high proportion of inpatients were prescribed antibiotics at the department level. This proportion was significantly higher in the NTH compared to the TH ( $85 \%$ vs $81 \%$ respectively, $\mathrm{p}<0.001)$. Both the duration of hospital stay and duration of antibiotic treatment were significantly longer in the TH (Table 1).

Overall, full-term baby delivered through normal vaginal delivery (FTND, ICD10- O 80.9, nonsurgical group) was the most commonly registered indications for hospital admission in both hospitals, 209/2539 (8\%) in the TH and 572/3019 (19\%) in the NTH. The next common indications were uterine prolapse (192, 8\%) and vaginal hysterectomy ( $\mathrm{VH}, 182,7 \%$, surgical group). At the NTH, surgical procedures such as lower segment cesarean section (LSCS, 480, 16\%) and abdominal hysterectomy ( $\mathrm{AH}, 328,11 \%)$ were the next most common indications. Also, 5\% in $\mathrm{TH}$ and $7 \%$ in NTH of the patients were admitted with indications of abortion or incomplete abortion without any other medical history, symptoms or indications.

Figure 1 Grouping of the inpatients based on recorded indications at the Obstetrics and Gynaecology Departments of two private a TH and NTH hospitals in Central India

Abbreviations: NTH= non-teaching hospital; OBGY= Obstetrics and gynaecology departments; $\mathrm{TH}=$ teaching hospital

Table 1 Patient characteristics and antibiotic prescribing in the Obstetrics and Gynaecology departments of two private hospitals in Central India 


\begin{tabular}{lll}
\hline & TH & NTH \\
\hline Total number of inpatients & 2539 & 3019 \\
Age; mean years (SD) & $34(13)^{*}$ & $30(11)$ \\
Patients prescribed AB; n (\%) & $2044(81)$ & $2567(85)^{*}$ \\
Duration of hospital stay; mean days (SD) & $8(9)^{*}$ & $3(3)$ \\
Duration of AB treatment; mean days (SD) & $8(7)^{*}$ & $5(3)$ \\
Total number of prescription days & 30616 & 12512 \\
\hline
\end{tabular}

Statistically significant values $(\mathrm{p}<0.001)$ are marked with *.

Abbreviations: $\mathrm{AB}=$ antibiotics, $\mathrm{NTH}=$ non-teaching hospital, $\mathrm{OBGY}=$ Obstetrics and gynaecology departments; $\mathrm{SD}=\mathrm{standard}$ deviation, $\mathrm{TH}=$ teaching hospital

The infectious and non-infectious categories

As explained in the method section, 5558 inpatients were divided into infectious (TH-230, NTH-175) and non-infectious (TH-2309, NTH-2844) categories and were further divided into surgical, nonsurgical and possible surgical groups (Figure 1 and Table 2). The number of prescriptions in both infectious and non-infectious categories in the TH was higher across all diagnoses groups than in the NTH. The hospital stay and duration of antibiotic treatment for the patients of the non-infectious category were significantly longer in the $\mathrm{TH}$ than in the NTH regardless if the surgery was performed or not. Moreover, in surgery groups in infections and non-infectious category in both hospitals, the number of patients received antibiotics for one day was less than $5 \%$. The length of antibiotic administration for one day can be a proxy for antibiotic prophylaxis, which is indicated for every surgical procedure (Table 2).

Seventy-six per cent of 405 inpatients in the infectious category were in the non-surgical group (TH161, and NTH-145), and 88\% (269/306) of those were prescribed antibiotics during a hospital stay. In the non-infectious category, 2204 patients appeared in the non-surgical group, (TH-829, and NTH-1375). In this group, more than $70 \%$ of inpatients received antibiotics during a hospital stay. The majority (98\%) of the inpatients in the possible surgery group had non-infectious indications, where $77 \%$ were prescribed antibiotics in the $\mathrm{TH}$ and $50 \%$ patients in the NTH.

\section{Antibiotic prescribing patterns in the categories and groups}

In both, infectious and non-infectious categories, across all three groups (surgical, non-surgical and possible surgical), use of generic names was more common, and adherence to the NLEMI was 
higher in the TH than in the NTH (Table 3).

In the infectious category, nitroimidazole derivatives (23\% and $24 \%)$ and tetracyclines (19\% and 16\%) were the most commonly prescribed in surgical and non-surgical groups at the $\mathrm{TH}$, respectively (Table 3). Overall, in this category, other beta-lactams (J01D) and FDCs were prescribed at a greater extent in the NTH than in the TH $(\mathrm{p}<0.001)$. In the surgical group of the infectious category, the FDCs of third-generation cephalosporins accounted for $53 \%$ of all prescriptions in the NTH while FDCs were not prescribed at all at the TH.

In the non-infectious category, third-generation cephalosporins and FDCs accounted for $58 \%$ of all prescriptions in the surgical group of the NTH whereas aminoglycosides (18\%), imidazole (17\%), and nitroimidazole derivatives (17\%) were most commonly prescribed in the TH. In the non-surgical group at the $\mathrm{TH}$, extended-spectrum penicillins and imidazole derivatives constituted of 52\% whereas at the NTH third-generation cephalosporins, and FDCs constituted 57\% of all prescriptions (Table 3).

Table 2 Demographic and prescription details of patients at category and group levels in the Obstetrics and Gynaecology departments of two private hospitals in Central India 
Infectious category

$\mathrm{N}=405(\mathrm{TH}=230, \mathrm{NTH}=175)$

\begin{tabular}{|c|c|c|c|c|c|c|}
\hline & \multicolumn{2}{|c|}{ Surgical $(n=89)$} & \multicolumn{2}{|c|}{$\begin{array}{l}\text { Non-surgical } \\
(n=306)\end{array}$} & \multirow{2}{*}{$\begin{array}{l}\text { Possible } \\
(n=10) \\
\mathrm{TH}\end{array}$} & \multirow{2}{*}{$\begin{array}{l}\text { Surgical } \\
\text { NTH }\end{array}$} \\
\hline & $\mathrm{TH}$ & NTH & $\mathrm{TH}$ & NTH & & \\
\hline \multirow[t]{2}{*}{ Total inpatients in each diagnosis group; $\mathrm{n}$} & 62 & 27 & 161 & 145 & 7 & 3 \\
\hline & & & & 126 & & \\
\hline Inpatients prescribed $\mathrm{AB} ; \mathrm{n}(\%)$ & $57(92)$ & $27(100)$ & $143(89)$ & $(87)$ & $6(86)$ & $3(100)$ \\
\hline Duration of hospital stay; mean days (SD) & $11(10)$ & $6(3)$ & $6(5) *$ & $3(3)$ & $13(5)$ & $3(2)$ \\
\hline Duration of $\mathrm{AB}$ treatment; mean days (SD) & $9(7)$ & $6(3)$ & $6(4)^{*}$ & $3(2)$ & $9(3)$ & $4(2)$ \\
\hline \multicolumn{7}{|l|}{$\mathrm{AB}$ prescriptions in each diagnosis group; } \\
\hline \multirow[t]{2}{*}{$\mathrm{n}$} & 974 & 186 & 1592 & 479 & 91 & 16 \\
\hline & & & 393 & & & \\
\hline $\mathrm{AB}$ prescriptions by generic name; $\mathrm{n}(\%)$ & $182(19)$ & $17(9)$ & $(25)^{*}$ & $15(3)$ & $2(2)$ & $0(0)$ \\
\hline Types of ABs substances prescribed; $n$ & 17 & 22 & 18 & 34 & 10 & 3 \\
\hline ABs prescribed using generic name; $\mathrm{n}(\%)$ & $7(41)$ & $2(9)$ & $8(44)$ & $3(9)$ & $1(10)$ & $0(0)$ \\
\hline Prescriptions of $\mathrm{ABs}$ found in the NLEMI; & & & 1084 & 271 & & \\
\hline $\mathrm{n}(\%)$ & $681(70)$ & $112(60)$ & $(68) *$ & $(57)$ & $54(59)$ & $3(19)$ \\
\hline \multicolumn{7}{|l|}{ Number of inpatients prescribed $A B s$ for } \\
\hline one day & $2(3)$ & $0(0)$ & $4(2)$ & $12(8)$ & $0(0)$ & $0(0)$ \\
\hline
\end{tabular}

Non-infectious category

$\mathrm{N}=5153(\mathrm{TH}=2309, \mathrm{NTH}=2844)$

\begin{tabular}{|c|c|c|c|c|c|c|}
\hline & \multicolumn{2}{|c|}{ Surgical $(\mathrm{n}=2506)$} & \multicolumn{2}{|c|}{$\begin{array}{l}\text { Non-surgical } \\
(n=2204)\end{array}$} & \multirow{2}{*}{$\begin{array}{l}\text { Possible } \\
(n=443)\end{array}$} & \multirow{2}{*}{$\begin{array}{l}\text { Surgical } \\
\text { NTH }\end{array}$} \\
\hline & TH & NTH & TH & NTH & & \\
\hline \multirow[t]{2}{*}{ Total inpatients in each diagnosis group; $\mathrm{n}$} & 1183 & 1323 & 829 & 1375 & 297 & 146 \\
\hline & 1030 & 1268 & & 1025 & & \\
\hline Inpatients prescribed $A B ; n(\%)$ & $(87)$ & $(95)^{*}$ & $592(71)$ & (75) & $216(73)$ & $119(82)$ \\
\hline Duration of hospital stay; mean days (SD) & $13(10)^{*}$ & $6(3)$ & $6(6) *$ & $3(3)$ & $10(9) *$ & $4(4)$ \\
\hline Duration of AB treatment; mean days (SD) & $9(8)^{*}$ & $6(3)$ & $6(4) *$ & $3(2)$ & $8(7)^{*}$ & $4(3)$ \\
\hline $\mathrm{AB}$ prescriptions in each diagnosis & 19024 & 7785 & 5165 & 3484 & 3770 & 562 \\
\hline
\end{tabular}


5757

$\mathrm{AB}$ prescriptions by generic name; $\mathrm{n}(\%)$

Types of ABs prescribed; $\mathrm{n}$

ABs prescribed using generic name; $\mathrm{n}(\%)$

Prescriptions of ABs found in the NLEMI;

n (\%)

(78)

(30)*

30

$12(43) *$

14920

3881

(50)

(82)*

$29(2)$

$72(5)$

$15(2)$

$111(8)$

$10(3)$

9 (6)

Statistically significant values $(\mathrm{p}<0.001)$ are marked with *. Abbreviations: $A B=$ antibiotics, NLEMI= National list of essential medicines India, $\mathrm{NTH}=$ non-teaching hospital, $\mathrm{OBGY}=$ Obstetrics and gynaecology departments, $\mathrm{SD}=$ standard deviation, $\mathrm{TH}=$ teaching hospital

Table 3 Classes and sub-classes of prescribed antibiotics at the category and group level in the Obstetrics and Gynaecology departments of two private hospitals in Central India 
Infectious $\mathrm{N}=(\mathrm{TH}-230, \mathrm{NTH}-175)$

\begin{tabular}{|c|c|c|c|c|c|c|}
\hline & \multicolumn{2}{|l|}{ Surgical } & \multicolumn{2}{|c|}{ Non-surgical } & \multicolumn{2}{|c|}{ Possible Surgical } \\
\hline & $T H$ & $N T H$ & $T H$ & $N T H$ & $T H$ & $N T H$ \\
\hline & $n(\%)$ & $n(\%)$ & $n(\%)$ & $n(\%)$ & $n(\%)$ & $n(\%)$ \\
\hline Total prescriptions & 974 & 186 & 1592 & 479 & 91 & 16 \\
\hline Tetracyclines; J01A, J01AA & $189(19)^{*}$ & $1(1)$ & $251(16)^{*}$ & $3(1)$ & $21(23)$ & - \\
\hline Beta-lactam ABs, penicillin; J01C & $83(9)$ & $20(11)$ & $237(15)$ & $69(14)$ & $7(8)$ & $3(19)$ \\
\hline Extended-spectrum penicillins; J01CA & $66(7)$ & - & $202(13)^{*}$ & $13(3)$ & $7(8)$ & - \\
\hline Comb. of penicillin incl. Beta-lactamase AB; J01CR & $17(2)^{*}$ & $20(11)$ & $29(2)^{*}$ & $56(12)$ & - & $3(19)$ \\
\hline Other Beta-lactam; J01D & $100(10)^{*}$ & $67(36)$ & $103(6)^{*}$ & $174(36)$ & $10(11)$ & - \\
\hline 3rd gen. cephalosporins; J01DD & $90(9)^{*}$ & $55(30)$ & $103(6)^{*}$ & $161(34)$ & $10(11)$ & - \\
\hline Aminoglycoside; J01G & $104(11)$ & $17(9)$ & $180(11)^{*}$ & $21(4)$ & - & - \\
\hline Other aminoglycosides; J01GB & $104(11)$ & $17(9)$ & $180(11)^{*}$ & $18(4)$ & - & - \\
\hline Quinolones; J01M, J01MA & $122(13)$ & $27(15)$ & $241(15)$ & $48(10)$ & $9(10)$ & - \\
\hline Fixed dose combination of ABs; J01R, J01RA* & - & $43(23)$ & - & $126(26)$ & - & $13(81)$ \\
\hline Other ABs; J01X & $129(13)$ & $9(5)$ & $183(11)^{*}$ & $20(4)$ & $2(2)$ & - \\
\hline Imidazole derivatives; J01XD & 129 (13) & $9(5)$ & $183(11)^{*}$ & $20(4)$ & $2(2)$ & - \\
\hline Nitroimidazole derivatives; $\mathrm{P} 01 \mathrm{~A}, \mathrm{P} 01 \mathrm{AB}$ & $224(23)^{*}$ & $2(1)$ & $390(24)^{*}$ & $4(1)$ & $30(33)$ & - \\
\hline
\end{tabular}

Non -infectious $\mathrm{N}=(\mathrm{TH}-2309, \mathrm{NTH}-2844)$

\begin{tabular}{|c|c|c|c|c|c|c|}
\hline & \multicolumn{2}{|l|}{ Surgical } & \multicolumn{2}{|c|}{ Non-surgical } & \multicolumn{2}{|c|}{ Possible Surgical } \\
\hline & $T H$ & $N T H$ & $T H$ & $N T H$ & $T H$ & $N T H$ \\
\hline & $n(\%)$ & $n(\%)$ & $n(\%)$ & $n(\%)$ & $n(\%)$ & $n(\%)$ \\
\hline Total prescriptions & 19024 & 7785 & 5165 & 3484 & 3770 & 562 \\
\hline Tetracyclines; J01A, J01AA & $1777(9)^{*}$ & $53(1)$ & $158(3)^{*}$ & $25(1)$ & $350(9)^{*}$ & $3(1)$ \\
\hline Beta-lactam ABs, penicillin; J01C & $2791(15)^{*}$ & $606(8)$ & $2156(42)^{*}$ & $543(15)$ & $400(11)$ & $48(9)$ \\
\hline Extended-spectrum penicillins; J01CA & $2466(13)^{*}$ & $61(1)$ & $1858(36)^{*}$ & $296(8)$ & $304(8)^{*}$ & $6(1)$ \\
\hline Comb. of penicillin incl. Beta-lactamase $A B ; J 01 C R$ & $279(1)^{*}$ & $545(7)$ & $290(6)$ & $247(7)$ & $93(2)^{*}$ & $42(7)$ \\
\hline Other Beta-lactam; J01D & $1316(7)^{*}$ & $2689(35)$ & $390(8)^{*}$ & $1410(40)$ & $478(13)^{*}$ & $182(32)$ \\
\hline 1stgen. cephalosporins; J01DB & $137(1)$ & $57(1)$ & $23(0)^{*}$ & $53(2)$ & $59(2)$ & $3(1)$ \\
\hline 2nd gen. cephalosporins; J01DC & $12(0)^{*}$ & $297(4)$ & - & $151(4)$ & $19(1)^{*}$ & $31(5)$ \\
\hline 3rd gen. cephalosporins; J01DD & $1167(6)^{*}$ & $2335(30)$ & $363(7)^{*}$ & $1193(34)$ & $400(11)^{*}$ & $148(26)$ \\
\hline
\end{tabular}




\begin{tabular}{|c|c|c|c|c|c|c|}
\hline Sulfonamide with timethoprime; J01E, J01EE & $131(1)^{*}$ & $8(0)$ & $20(0)$ & $10(0)$ & $46(1)$ & - \\
\hline Macrolides, lincosamides J01F & $7(0)^{*}$ & $34(0)$ & $12(0)^{*}$ & $34(1)$ & $18(1)$ & - \\
\hline Macrolides; J01FA & $7(0)^{*}$ & $23(0)$ & $5(0)^{*}$ & $29(1)$ & $9(0)$ & - \\
\hline Aminoglycoside; J01G & $3379(18)^{*}$ & $528(7)$ & $734(14)^{*}$ & $156(4)$ & $694(18)^{*}$ & $58(10)$ \\
\hline Other aminoglycosides; J01GB & $3379(18)^{*}$ & $522(7)$ & $734(14)^{*}$ & $156(4)$ & $694(18)^{*}$ & $58(10)$ \\
\hline Quinolones; J01M, J01MA & $3071(16)$ & $1317(17)$ & $432(8)^{*}$ & $403(11)$ & $544(14)$ & $64(11)$ \\
\hline Fixed dose combination of ABs; J01R, J01RA* & $17(0)^{*}$ & $2217(28)$ & - & $801(23)$ & $2(0)^{*}$ & $159(28)$ \\
\hline Other ABs; J01X & $3364(18)^{*}$ & $300(4)$ & $804(16)^{*}$ & $100(3)$ & $645(17)^{*}$ & $45(8)$ \\
\hline Imidazole derivatives; J01XD & $3320(17)^{*}$ & $300(4)$ & $804(16)^{*}$ & $100(3)$ & $636(17)^{*}$ & $40(7)$ \\
\hline Drugs for treatment of tuberculosis; J04A, J04AM & - & $3(0)$ & - & - & - & $3(1)$ \\
\hline Nitroimidazole derivatives; $\mathrm{P} 01 \mathrm{~A}, \mathrm{P} 01 \mathrm{AB}$ & $3162(17)^{*}$ & $30(0)$ & $459(9)^{*}$ & $10(0)$ & $593(16)$ & - \\
\hline
\end{tabular}

Statistically significant values $(\mathrm{p}<0.001)$ are marked with *. Abbreviations: $A B=$ antibiotics, ATC=The Anatomical Therapeutic Chemical Classification System, $\mathrm{n}=$ number of prescriptions, NTH=non-teaching hospital, OBGY= Obstetrics and Gynaecology departments, TH= teaching hospital

\section{Antibiotic prescribing in DDD/1000 patients}

DDD per 1000 patients was calculated at the substance level of ATC. It is presented for DU90\% of the prescribed antibiotics for each diagnoses group (Table 4). Overall, higher DDD/1000 patients were prescribed in the $\mathrm{TH}$ compared to the NTH in both categories. In the TH, regardless of the presence of an infectious indication, doxycycline (in surgical and possible surgical group) and ampicillin (in the non-surgical group) the prescribed DDD/1000 patients was highest. In the NTH in the infectious category, cefixime was most commonly prescribed in the surgical group, ceftriaxone in the non-surgical group and metronidazole in the possible surgical group, whereas in noninfectious category cefixime was most frequently prescribed (Table 4).

Table 4 DDD/1000 patients for the most prescribed antibiotic substances (DU90\%) at category and group level in the Obstetrics and Gynaecology departments of two private hospitals in Central India 


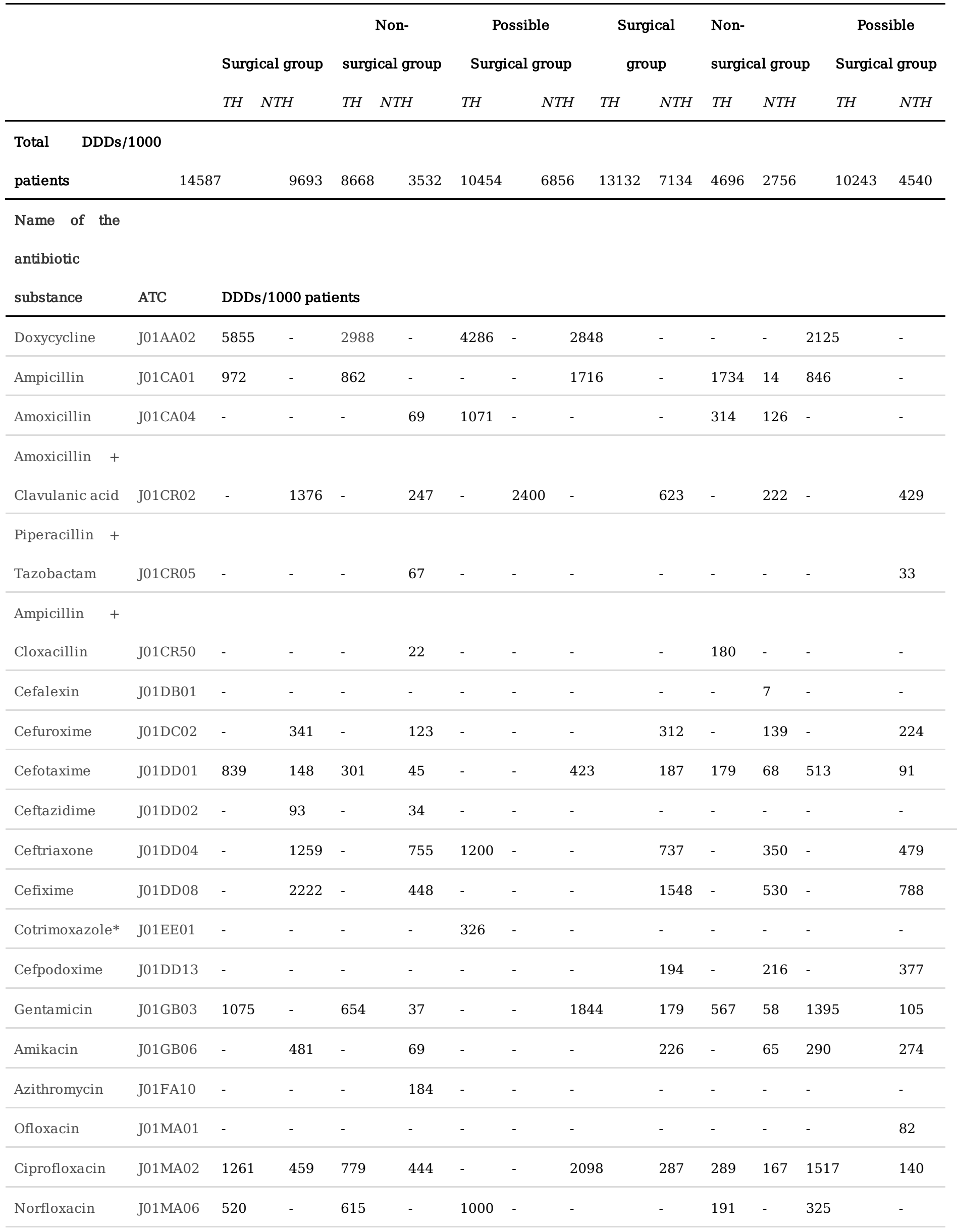




\begin{tabular}{|c|c|c|c|c|c|c|c|c|c|c|c|c|c|}
\hline Levofloxacin & J01MA12 & - & 1111 & - & 131 & - & - & - & 1083 & - & 174 & - & 340 \\
\hline Moxifloxacin & J01MA14 & - & - & - & - & - & - & - & 118 & - & - & - & 60 \\
\hline \multicolumn{14}{|l|}{ Ceftazidime +} \\
\hline Tazobactam & J01RA*82 & - & - & - & - & - & - & - & - & - & - & - & 34 \\
\hline \multicolumn{14}{|l|}{ Ciprofloxacin + } \\
\hline Tinidazole & J01RA*73 & - & - & - & 19 & - & - & - & - & - & - & - & - \\
\hline \multicolumn{14}{|l|}{ Cefoperazone } \\
\hline + Sulbactam & J01RA*83 & - & 259 & - & 30 & - & - & - & 56 & - & 16 & - & 159 \\
\hline \multicolumn{14}{|l|}{ Ceftriaxone +} \\
\hline Sulbactam & J01RA*84 & - & 315 & - & 159 & - & - & - & 177 & - & 63 & - & 253 \\
\hline \multicolumn{14}{|l|}{ Ceftriaxone +} \\
\hline Tazobactam & J01RA*85 & - & 537 & - & 352 & - & 2000 & - & 916 & - & 242 & - & 392 \\
\hline \multicolumn{14}{|l|}{ Cefotaxime +} \\
\hline Sulbactam & J01RA*86 & - & - & - & 193 & - & - & - & 278 & - & 120 & - & 41 \\
\hline \multicolumn{14}{|l|}{ Cefixime $\quad+$} \\
\hline Ofloxacin & J01RA*91 & - & 833 & - & - & - & - & - & - & - & 127 & - & - \\
\hline \multicolumn{14}{|l|}{ Ofloxacin + } \\
\hline Metronidazole & J01RA*72 & - & - & - & - & - & 2456 & - & 82 & - & - & - & 87 \\
\hline \multicolumn{14}{|l|}{ Metronidazole } \\
\hline (paraenteral) & J01XD01 & 1960 & 259 & 1088 & 104 & - & - & 2643 & 131 & 911 & 52 & 2066 & 152 \\
\hline \multicolumn{14}{|l|}{ Metronidazole } \\
\hline (oral) & P01AB01 & 2105 & - & 1381 & - & 2571 & - & 1560 & - & 331 & - & 1166 & - \\
\hline
\end{tabular}

\footnotetext{
* Sulfamethoxazole + trimethoprim, Abbreviations: ATC= Anatomical Therapeutic Chemical Classification code, DDD= Defined Daily Dose, J01RA*= ATC codes according to Sharma et al. (17), NTH= Non-teaching hospital, OBGY= Obstetrics and gynaecology departments, TH= Teaching hospital
}

\section{Discussion}

To the best of our knowledge, this is a first study that describes and compares antibiotic prescribing in relation to the presence of infection and surgery conducted among the inpatients at OBGY 
departments of two private sector hospitals in India. In the present study, the patients were categorized by clinical presence and absence of infectious indications and were further divided into surgical, non-surgical and possible surgical groups. Although few studies conducted at hospital level or for the shorter period, are published $(3,16,17)$, none of them has shown the prescribing patterns in OBGY department for the stated categories and groups. It is thus, clear that there is a limitation to compare the results of the present study with other studies.

Our results show an overall high prescribing rate of antibiotics to the inpatients, where more than $80 \%$ received antibiotics during their hospital stay. This percentage of the antibiotic prescribing rate in the OBGY department was comparable to a study conducted by Sharma et al., (86\%) (17) but was lower compared than the study conducted by Alvarez et al. in a rural hospital in Andhra Pradesh, India (92\%) (3). In both studies, the analysis was conducted for all admitted patients but did not correspond to the indications. In the present study, we analyzed data corresponding to recorded indications at both hospitals and observed extensive prescribing of antibiotics to the patients without any record of infectious indication. However, the antibiotics were prescribed to a higher extent to the patients who underwent surgery (>85\%) than in the nonsurgical group (>70\%) in our study. Prescribing antibiotics to the patients who did not undergo surgery or had no signs of infection could not be explained at present and need further study.

\section{Adherence to guidelines}

Local prescribing guidelines were not present in either of the hospitals. The prescribing patterns were compared with the national and WHO list of essential medicines. The prescriptions in the $\mathrm{TH}$ showed significantly higher adherence to the NLEMI and prescribing by generic name than the NTH. Higher adherence to the national guidelines, not prescribing FDCs and more use of generic names in the infectious category was also observed in the TH than in the NTH. Studies show that the medical representatives lure consultants to prescribe antibiotics by trade name instead of generic name favouring the sale of the products of a particular pharmaceutical company (25). Consultants that work in the NTH are allowed to run private practices, where they were free to meet the medical representatives of pharmaceutical companies. These meetings are more likely to influence the contents of a doctor's prescription. However, at the TH as per the hospital policy, generic medicines were purchased by the management and supplied to the pharmacy. Also, the consultants were not allowed to run a private practice, and contacts with medical representatives 
were also restricted in the TH. This could perhaps be, one of the explanations for the observed trade name prescribing patterns in the NTH.

Also, this might be a positive impact of continuing medical education seminars held at the $\mathrm{TH}$ as a part of the routine academic activity, might also have motivated towards being adherent to the recommendations. These hospital policies could be modified contextually and implemented at other similar settings to rationalise prescribing of antibiotics.

\section{Hospitalisation and antibiotic treatment duration and treatment costs}

Patients admitted to the $\mathrm{TH}$ had both; longer hospital stay and duration of antibiotic treatment significantly, compared to the patients in the NTH (Table 1). These long duration of stay and antibiotic prescribing days are a risk factor for HAIs. Suspicion or presence of HAIs results in prescribing antibiotics (26). Data was not available on the prevalence of HAIs from the study hospitals for entire study duration, due to reduced utilisation of diagnostic facilities. Therefore, it will be inappropriate to comment on the relationship between HAIs and antibiotic prescribing. However, the HAI prevalence could be expected to be similar to other comparable settings (27).

Stay days are directly related to receiving more prescriptions and increased treatment costs, but at the $\mathrm{TH}$, all patient services, including stay, were free, so patients might not have enforced for early discharge. While in the NTH patients paid out-of-pocket for medical services and might have compelled the patients to choose for an early discharge.

\section{Antibiotic prescribing in infectious and non-infectious categories}

Choice of the drugs both at the category and group level varied significantly between the hospitals. The FDCs (J01RA*) and third-generation cephalosporins were frequently prescribed at the NTH, constituting more than half prescriptions. These FDCs are neither enlisted in the WHO list of essential medicines nor the NLEMI (17). Combining antibiotic substances to prepare an FDC is often irrational and costlier $(1,28)(17)$. Prescribing a broad spectrum and FDCs results in unnecessary exposure to antibiotics and increase the risk of bacterial resistance. This issue needs immediate attention of the policymakers and the prescribers $(17,29)$.

Presence of bacterial infection is an appropriate indication for antibiotic treatment in post-operative procedures, whereas a surgery per se is an indication to receive a single prophylactic dose of 
antibiotic before or during the surgery. In our study, contrary to the recommendations, antibiotics were prescribed even in the absence of any clinical decision or laboratory confirmation for the presence of infection. More than 70\% of patients in the non-infectious, non-surgical group were also prescribed antibiotics at both hospitals (Table 2). These results are comparable with a study at another Indian hospital where nearly $70 \%$ of women with severe pre-eclampsia or eclampsia, which is not an indication for antibiotic use, were prescribed antibiotics (30). Most of these women had LSCS (54\%). Prescribing prophylactic antibiotics for surgeries such as LSCS is recommended while in our study, only $23 \%$ of patients had LSCS (30). Prescribing antibiotic treatment for the noninfectious, non-surgical cases is unindicated and point towards the risk of development of antibiotic resistance.

\section{Antibiotic prescribing in the groups}

Our results from the surgical groups show that a large proportion of the inpatients, not having a confirmed infection, received antibiotic prescriptions. Despite evidence and recommendations for prescribing a single prophylactic dose of an antibiotic for the surgical indications. Moreover, the duration of prescribing antibiotics exceeded recommended prophylactic duration among almost all inpatients in the surgery groups of both categories $(31,32)$. Prescribing antibiotics as treatment is only indicated in cases of a postoperative or simultaneous infection for procedures such as cesarean section and hysterectomies (32). Comparable prescribing patterns have previously been presented in two Indian studies; by Sharma et al. and Kumar et al.; where patients were treated with antibiotic therapy for multiple days after cesarean section instead of single-dose prophylaxis (30, 33). Prescribing extended duration of antibiotics to non-surgical patients not only risk to the development of antibiotic resistance but increase the treatment cost.

A survey conducted among 650 surgeons in India reflected that surgeons do prescribe antibiotics for extended periods than recommended in the standard surgeon's guidelines and recommendations (34). Lack of local prescribing guidelines could be one of the underlying reasons for the observed high antibiotic prescribing. Interventions such as development and successful implementation of relevant antibiotic prophylaxis guidelines and regular prescription audits would help to reduce antibiotic prescribing, as demonstrated for LSCS patients in a single centre study from Serbia (35). The Siberian research shows a significant post-intervention reduction in the use of $3^{\text {rd }}$ generation cephalosporins and the total treatment cost by $47 \%$ (35). Reduced motivation to maintain personal hygiene is another common factor influencing antibiotic prescribing in LMICs. 
However, precise underlying reasons for prescribing antibiotics to the extended duration for unindicated conditions in the present study settings need further investigation using a qualitative approach.

In the non-surgical groups at both hospitals, FTND was the most common condition for admission and majority of the patients who gave birth to FTND also received antibiotic treatment. Antibiotic prescription to this group of patients cannot be justified as even the prophylactic use of antibiotics for FTND is not recommended (36). A previous study from Ujjain district in India has shown an antibiotic prescribing to 87\% FTND patients (37). A high proportion of patients in this group received antibiotic treatment is another highlight. Although, an episiotomy is a possible indication for antibiotic prophylaxis in FTND, however, according to a Cochrane review, further studies are required to ascertain the need for antibiotic prophylaxis for an episiotomy (38). Thus, a focused study is suggested to investigate the rationality of prescribing antibiotics for FTND.

We also found that in both hospitals, at the time of admissions, a considerable proportion of the inpatients appeared with the indications that led to the abortions. Reasons for part of these admissions could be linked to the self-induced abortions. Some of these admissions might also be due to the spontaneous and clinically induced abortions due to abnormal growth of the fetus, such as intrauterine growth restriction of the fetus. However, further detail investigations are needed in the settings to confirm these assumptions.

Future implications

- Feedback to the prescribers on the current situation in OBGY departments

- Qualitative studies to explain the underlying reason for antibiotic prescribing for non-indicated conditions and for a longer time than recommended

- Emphasis on infection prevention through motivating hygiene in hospital and community

- Continue prescription follow up and audits

\section{Strengths and limitations}

The major strength of this study is the relatively large population of 5558 patients included in the analysis. The data was collected over three years, that contributed to study the effect of seasonal variations on prescribing of the antibiotics. 
Additionally, the data was collected comprehensively to overcome selection bias. All diagnoses were checked manually from records and patients were divided into diagnosis groups in consultation with two local obstetricians and gynaecologists for comprehensive categorisation of patients in the diagnoses groups and subgroups. The consultants were not identified at any stage of the study. This method of data collection might have given freedom of deciding the treatment plan for the patients.

Our study also had a few limitations. Firstly, since the data was collected manually, the possibility of missing data was foreseen. For the missing data, the records in the archive were checked before analysis. For a small proportion of patients, information on whether the patients were operated or not, could not be retrieved. For such patient records, a third diagnosis group, "possible surgical" was created to nullify the probable overestimation of antibiotic prescribing, otherwise. The diagnoses (indications) were not validated externally, as this was not the aim of the study. The use of DDD has been criticised because it has shown a weak correlation with prescribed daily doses in some settings. However, prescribed daily doses may vary among healthcare facilities, and DDD allows comparison among hospitals or clinics even when prescribed daily doses are different. The absence of computerised record systems in hospitals, and personal identification numbers, unexperienced staff for data collection and high staff turnover make a comprehensive study like this; time-consuming and tedious, that causes a delay in the analysis. We are aware that extensive manual checking and adding the ICD codes, and ATC codes for the new FDCs in the data have prolonged the analysis and delayed the presentation. However, the use of human resources is the only option to conduct such detailed studies at resource-constrained settings and at the same time, leads to a more accurate description of the prescribing patterns.

\section{Conclusions}

In general, antibiotic prescribing was high in OBGY departments at both hospitals; however, more common in the NTH than in the TH. Antibiotics were empirically prescribed to the patients without having any clinical indications of infections at both the hospitals. Patients that underwent elective surgeries received antibiotic treatment for multiple days, despite recommendations of single-dose prophylaxis. Broad-spectrum antibiotics, including new FDCs, were more frequently prescribed by trade names at the NTH compared to the TH.

Multiple-step approach in terms of antibiotic stewardship program is suggested to address the issue of over-prescribing of the antibiotics. The program could include; presentation of the study results as feedback to the consultants, the involvement of the consultants in the development of local 
diagnose-specific antibiotic prescribing guidelines, implementation of the guidelines and, periodic training and educational sessions. The antibiotic stewardship needs to be accompanied by continuous surveillance of the prescriptions and qualitative studies to explore the underlying reasons for the present prescribing patterns.

\title{
Abbreviations
}

\author{
AH- Abdominal hysterectomy \\ ATC- Anatomical Therapeutic Chemical \\ DDD- Defined Daily Doses
}

FDCs- Fixed-dose combinations

FTND- Full-term normal delivery

HAIs- Healthcare-associated infections

ICD 10- International Statistical Classification of Diseases and Related Health Problems- Tenth Revision

LCSC- Lower segment caesarian section

LMICs- Low and middle- income countries

NLEMI- National List of Essential Medicines of India

NTH- Non-teaching hospital

OBGY- Obstetrics and Gynecology

TH- Teaching hospital

WHO- World Health Organization

WHOCC- WHO Collaborating Centre for Drug Statistics Methodology

\section{Declarations}

\section{Ethics approval and consent to participate}

The ethics committee of Ruxmaniben Deepchand Gardi Medical College, Ujjain, approved the study with approval number: 41/2007 and 114/2010. This was an observational study where the data 
collection was done using patient files. The study did not interfere with the patients receiving the treatment, and none of the patients was contacted during the study period. Each patient was given a unique code locally. The analysis was performed in groups using these unique codes to maintain confidentiality. No exclusions were made by age, sex or other demographic criteria.

\section{Consent for publication}

Not applicable

\section{Availability of data and material}

The data will be made available to all interested researchers upon request made to; The Chairman, Ethics Committee, R. D. Gardi Medical College, Agar Road, Ujjain, Madhya Pradesh, India 456006 (Email: iecrdgmc@yahoo.in, uctharc@bsnl.in), giving all details of the article. The ethical approval number: 41/ 2007 and 114/2010 to be quoted along with the request.

\section{Competing interests}

The authors declare that they have no competing interests

Funding Swedish Research Council (K2007-70X-20514-01-3, K2010-

396 70X-20514-04-3, 2017-01327) and Asia Link (348-2006-6633). MS is the recipient of Erasmus Mundus Lot-15.

\section{Authors' contributions}

AM, MS, FJ analysed the data. AM, MS, and FJ interpreted and presented the data and were responsible for drafting the manuscript. CSL and MS gave critical suggestions for the manuscript. All authors read and approved the final manuscript.

\section{Acknowledgements}

The authors extend thanks to the nursing staff of both hospitals involved in the data collection, to the management of both hospitals, to the consultants of the OBGY departments and Dr V.K. Mahadik (Medical Director) for the permission and support during the study.

\section{References}


1. Organization WH. The evolving threat of antimicrobial resistance: options for action: World Health Organization; 2012.

2. Westly E. India moves to tackle antibiotic resistance. Nature. 2012;489(7415):192.

3. Alvarez-Uria G, Zachariah S, Thomas D. High prescription of antimicrobials in a rural district hospital in India. Pharm Pract (Granada). 2014;12(2):384.

4. Morgan DJ, Okeke IN, Laxminarayan R, Perencevich EN, Weisenberg S. Non-prescription antimicrobial use worldwide: a systematic review. Lancet Infect Dis. 2011;11(9):692-701.

5. Kotwani A, Holloway K. Access to antibiotics in New Delhi, India: implications for antibiotic policy. J Pharm Policy Pract. 2013;6:6.

6. Laxminarayan R, Heymann DL. Challenges of drug resistance in the developing world. BMJ. 2012;344:e1567.

7. Bell BG, Schellevis F, Stobberingh E, Goossens H, Pringle M. A systematic review and metaanalysis of the effects of antibiotic consumption on antibiotic resistance. BMC Infect Dis. 2014;14:13.

8. Bronzwaer SL, Cars O, Buchholz U, Molstad S, Goettsch W, Veldhuijzen IK, et al. A European study on the relationship between antimicrobial use and antimicrobial resistance. Emerg Infect Dis. 2002;8(3):278-82.

9. J. ON. Antimicrobial resistance: tackling a crisis for the health and wealth of nations, The review on antimicrobial resistance. 2014.

10. Okeke IN, Laxminarayan R, Bhutta ZA, Duse AG, Jenkins P, O'Brien TF, et al. Antimicrobial resistance in developing countries. Part I: recent trends and current status. Lancet Infect Dis. 2005;5(8):481-93.

11. Organization WH. Global Action Plan on Antimicrobial Resistance. 2015.

12. Van Boeckel TP, Gandra S, Ashok A, Caudron Q, Grenfell BT, Levin SA, et al. Global antibiotic consumption 2000 to 2010: an analysis of national pharmaceutical sales data. Lancet Infect Dis. 2014;14(8):742-50.

13. Deshpande K, Ravi Shankar, Diwan V, Lonnroth K, Mahadik VK, Chandorkar RK. Spatial pattern of private health care provision in Ujjain, India: a provider survey processed and analysed with a Geographical Information System. Health Policy. 2004;68(2):211-22.

14. Organization WH. Health statistics 2009 [Available from:

http://who.int/whosis/whostat/EN_WHS09_Full.pdf.

15. P C. Healthcare in India: Features of one of the most privatised systems in the world; 1 September 2009. 
16. Kumar R, Indira K, Rizvi A, Rizvi T, Jeyaseelan L. Antibiotic prescribing practices in primary and secondary health care facilities in Uttar Pradesh, India. J Clin Pharm Ther. 2008;33(6):625-34.

17. Sharma M, Eriksson B, Marrone G, Dhaneria S, Lundborg CS. Antibiotic prescribing in two private sector hospitals; one teaching and one non-teaching: a cross-sectional study in Ujjain, India. BMC Infect Dis. 2012;12:155.

18. Kumar SG, Adithan C, Harish BN, Sujatha S, Roy G, Malini A. Antimicrobial resistance in India: A review. J Nat Sci Biol Med. 2013;4(2):286-91.

19. Organization WH. World Health Statistics. 2014.

20. John J. IAP Policy on Age of Children for Pediatric Care. Indian Pedciatrics. 1999;36:461-3.

21. Organization WH. Introduction to Drug Utilization Research. 2003.

22. Organization WH. International Statistical Classificatoion of Disease and Related Health Problems - 10th revision. 2010.

23. Organization WH. Collaborating centre for drug statistics methodology, anatomical therapeutic chemical classification with defined daily doses 2017 [Available from: https://www.whocc.no/atc_ddd_index/.

24. Directorate General of Health Services MoHaFW, Government of India. National List of Essential Medicines 2011 [Available from: https://www.nhp.gov.in/national-list-of-essentialmedicines-2011_pg.

25. Blumenthal D. Doctors and drug companies. N Engl J Med. 2004;351(18):1885-90.

26. Saviteer SM, Samsa GP, Rutala WA. Nosocomial infections in the elderly. Increased risk per hospital day. Am J Med. 1988;84(4):661-6.

27. Pathak A, Saliba EA, Sharma S, Mahadik VK, Shah H, Lundborg CS. Incidence and factors associated with surgical site infections in a teaching hospital in Ujjain, India. Am J Infect Control. 2014;42(1):e11-5.

28. Organization WH. Global Strategy for Containment of Antimicrobial Resistance. 2001.

29. Sharma M, Damlin AL, Sharma A, Stalsby Lundborg C. Antibiotic prescribing in medical intensive care units--a comparison between two private sector hospitals in Central India. Infect Dis (Lond). 2015;47(5):302-9.

30. Kumar S, Bansal D, Hota D, Jain M, Singh P, Pandey BL. Assessment of clinical outcomes and prescribing behavior among inpatients with severe preeclampsia and eclampsia: an Indian experience. Indian J Pharmacol. 2014;46(1):18-23.

31. Smaill FM, Grivell RM. Antibiotic prophylaxis versus no prophylaxis for preventing infection after cesarean section. Cochrane Database Syst Rev. 2014(10):CD007482. 
32. van Schalkwyk J, Van Eyk N, Society of O, Gynaecologists of Canada Infectious Diseases C. Antibiotic prophylaxis in obstetric procedures. J Obstet Gynaecol Can. 2010;32(9):878-92.

33. Sharma JB, Gupta N, Aggarwal P, Mittal S. A survey of obstetricians' practice of using prophylactic antibiotics in vaginal deliveries and caesarean sections. J Indian Med Assoc. 2008;106(3):147-9.

34. Kulkarni Ra KR, Kochhar P, Dargude V, Rajadhyakshya S, et al. Patterns of antimicrobial use by surgeons in India. Indian J Surg 2005;67(6):308-15.

35. Ristic S, Miljkovic B, Vezmar S, Stanojevic D. Are local clinical guidelines useful in promoting rational use of antibiotic prophylaxis in caesarean delivery? Pharm World Sci. 2010;32(2):139-45.

36. Liabsuetrakul T, Lumbiganon P, Mori R, Gulmezoglu M, Souza JP, Maternal WHOGSo, et al. A secondary analysis of the WHO Global Survey on Maternal and Perinatal Health for antibiotics used in vaginal deliveries. Int J Gynaecol Obstet. 2014;124(3):240-3.

37. Sharma M, Sanneving L, Mahadik K, Santacatterina M, Dhaneria S, Stålsby Lundborg C. Antibiotic prescribing in women during and after delivery in a non-teaching, tertiary care hospital in Ujjain, India: a prospective cross-sectional study. J Pharm Policy Pract. 2013;6:9.

38. Liabsuetrakul T, Choobun T, Peeyananjarassri K, Islam M. Antibiotic prophylaxis for operative vaginal delivery. Cochrane Database Syst Rev. 2004(3):CD004455.

\section{Figures}




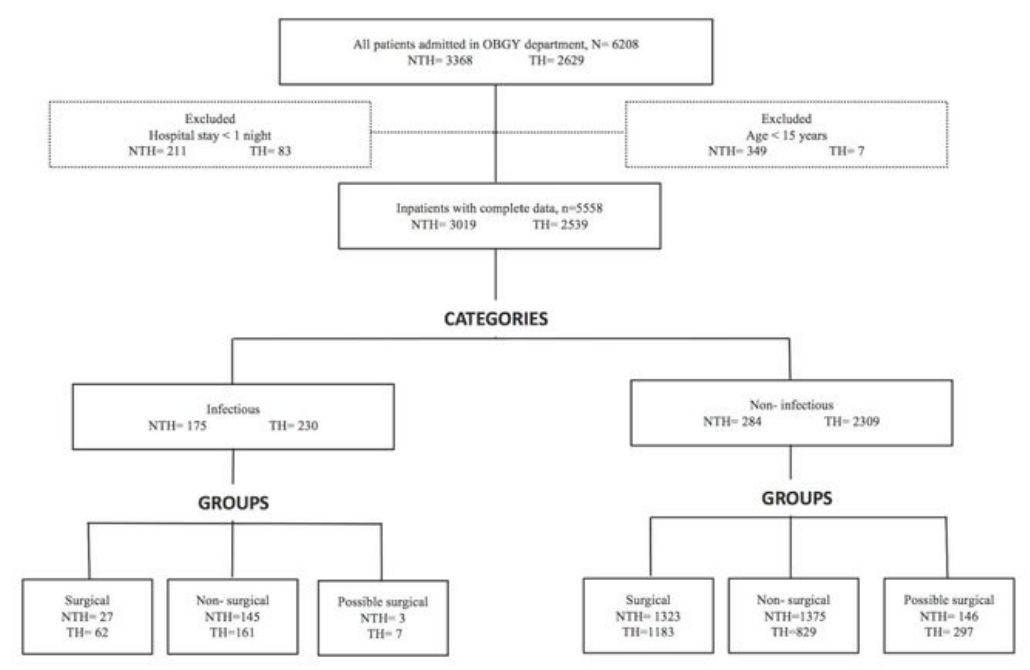

\section{Figure 1}

Grouping of the inpatients based on recorded indications at the Obstetrics and Gynaecology Departments of two private a TH and NTH hospitals in Central India Abbreviations: NTH= non-teaching hospital; OBGY= Obstetrics and gynaecology departments; $\mathrm{TH}=$ teaching hospital 